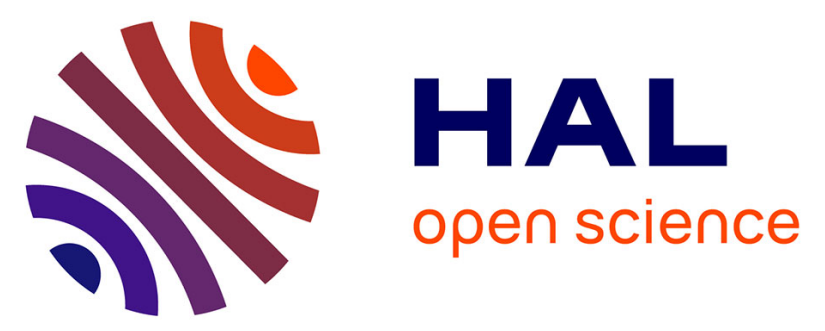

\title{
Encapsulation effect of $\pi$-conjugated quaterthiophene on the radial breathing and tangential modes of semiconducting and metallic single-walled carbon nanotubes
}

Jamal Chenouf, Mourad Boutahir, B. Fakrach, A. Rahmani, H. Chadli, Patrick Hermet, Jose Mejía-lópez, Abdelali Rahmani

\section{To cite this version:}

Jamal Chenouf, Mourad Boutahir, B. Fakrach, A. Rahmani, H. Chadli, et al.. Encapsulation effect of $\pi$-conjugated quaterthiophene on the radial breathing and tangential modes of semiconducting and metallic single-walled carbon nanotubes. Journal of Computational Chemistry, 2020, 41 (28), pp.2420-2428. 10.1002/jcc.26408 . hal-03017613

\section{HAL Id: hal-03017613 https://hal.science/hal-03017613}

Submitted on 21 Nov 2020

HAL is a multi-disciplinary open access archive for the deposit and dissemination of scientific research documents, whether they are published or not. The documents may come from teaching and research institutions in France or abroad, or from public or private research centers.
L'archive ouverte pluridisciplinaire HAL, est destinée au dépôt et à la diffusion de documents scientifiques de niveau recherche, publiés ou non, émanant des établissements d'enseignement et de recherche français ou étrangers, des laboratoires publics ou privés. 


\title{
Encapsulation effect of $\pi$-conjugated
} quaterthiophene on the Radial breathing and tangential modes of semiconducting and metallic single-walled carbon nanotubes

\author{
Chenouf J. ${ }^{1}$ | Boutahir M. $^{2 *, 1}$ | Fakrach B. ${ }^{3,1}$ | \\ Rahmani AH. $^{1}$ | Chadli H. ${ }^{1}$ | Patrick Hermet $^{4}$ | \\ Mejía-López J. ${ }^{2} \quad$ Rahmani A. ${ }^{1}$
}

\footnotetext{
${ }^{1}$ Laboratoire d'Etude des Matériaux Avancés et Applications (LEM2A), Université Moulay Ismail,FSM-ESTM, BP 11201, Zitoune, 50000 Meknes, Morocco

${ }^{2}$ Centro de Investigación en Nanotecnología y Materiales Avanzados CIEN-UC, Facultad de Física, Pontificia Universidad Católica de Chile. Cedenna, Santiago, Chile

${ }^{3}$ Laboratoire de Physique Théorique et Appliquée, Université Sidi Mohammed Ben Abdellah, Faculté des Sciences Dhar El Mahraz Fez, Morocco

${ }^{4}$ Institut Charles Gerhardt Montpellier, Univ. Montpellier, CNRS, ENSCM, Montpellier, France
}

\section{Correspondence}

Mourad Boutahir, Centro de Investigación en Nanotecnología y Materiales Avanzados CIEN-UC, Facultad de Física, Pontificia Universidad Católica de Chile. Cedenna, Santiago, Chile

Email: mourad.boutahir@fis.puc.cl

\section{Funding information}

CONICYT Posdoctodado 2020, Grant/Award Number: 3200046; Financiamiento basal para centros científicos y tecnológicos de excelencia, Grant/Award Number: AFB180001
We developed a hybrid approach, combining the density functional theory, molecular mechanics, bond polarizability model and the spectral moment's method to compute the nonresonant Raman spectra of a single quaterthiophene (4T) molecule encapsulated into a single-walled carbon nanotube (metallic or semiconducting). We reported the optimal tube diameter allowing the 4T encapsulation. The influence of the encapsulation on the Raman modes of the $4 \mathrm{~T}$ molecule and those of the nanotube (radial breathing modes and tangential modes) are analyzed. An eventual charge transfer between the $4 \mathrm{~T}$ oligomer and the nanotube is discussed.

\section{K E Y W O R D S}

Thiophene; CNTs; Raman spectroscopy; Charge transfer 


\section{1 | INTRODUCTION}

Organic solar cells have received a lot of attention in recent years because they make it possible to diversify renewable energy sources thanks to their simple preparation, lightweight, low technological cost and large-area flexible fabrication ${ }^{1 ; 2 ; 3 ; 4 ; 5}$. However, their energy efficiency and durability need to be improved for the development of sustainable technology. In these devices, photovoltaic conversion is based on the separation of photogenerated charges at an interface between electron donor and acceptor materials, which imposes some constraints on the photoactive layer of the cells.

Fullerene derivatives are among the most popular materials used in organic or related photovoltaic devices $6 ; 7 ; 8 ; 9 ; 10 ; 11$. In particular, organic photovoltaic (OPV) solar cells using a bulk-heterojunction (BHJ) of conjugated polymer donors and fullerene derivative acceptors have shown the best performance with a solar power conversion efficiency (PCE) of $11.5 \%^{12 ; 13}$. Single-walled carbon nanotubes (SWCNT) are also interesting potential candidates to replace the electronaccepting and transport fullerene phase in BHJ OPV systems ${ }^{14 ; 15}$. Indeed, carbon nanotubes (CNTs) offer distinctive properties that are not found in fullerenes, such as: (i) the very broad spectral coverage with band-gap electronic transitions well into the near-IR spectral region to match the solar spectrum ${ }^{16}$, (ii) a charge mobility (about $105 \mathrm{~cm}^{2} / \mathrm{V} . \mathrm{s}$ ) ${ }^{17}$ that may facilitate charge carrier transfer and/or dissociation and transport over an extended distance because of their large surface area (above $400 \mathrm{~m}^{2} / \mathrm{g}$ ) and (iii) electron-accepting properties ${ }^{18 ; 19}$.

Despite the fact that graphene has emerged to become the center of attention in the field of carbon nanomaterials in recent years ${ }^{20}$, there are still distinct advantages for the use of CNTs in energy conversion applications. Several studies on blends of conjugated polymers and SWCNT suggest that efficient exciton quenching occurs at these interfaces and many studies have shown direct evidence for the generation of mobile carriers in polymer-SWCNT composites ${ }^{21 ; 22 ; 23 ; 24}$. After exciton dissociation, ballistic carrier transport in single tubes and extremely high conductivity in networks suggest that SWCNT may yield higher mobilities and form more conductive percolation networks than fullerenes. OPV cells based on CNTs as acceptor on the concept of BHJ is one of the most promising. Nevertheless, the efficiency of this kind of device is limited because of the incomplete exciton dissociation ${ }^{25 ; 26}$. In addition, the electronic diversity of nanotubes produced which are typically a mixture of metallic and semiconducting $\mathrm{CNTs}^{27 ; 28}$ may affect the device efficiency due to the presence of the amount of metallic CNTs. As metallic SWCNTs do not have a true bandgap, one would naturally suspect these species to act as charge carrier or exciton recombination centers, lowering the efficiency of charge separation, and, by extension, the efficiency of devices. Thus, only semiconducting SWCNT are interesting for OPV as different authors report three-times long-lived charge carriers for the photocurrent generation by eliminating metallic CNTs ${ }^{29 ; 30}$. Exciton separation across this interface could be possible, but it has been revealed that energy transfer rates limiting the ability of the Donor/Acceptor interface to separate excitons in free charge carriers, decrease with the diameter of the tubes ${ }^{31}$. A stable structure of the hybrid system with an optimal diameter to decrease energy transfer and allows the exciton separation at the interface is principal.

The inferior OPV device stability still remains a great challenge. Many factors limit the stability of organic solar cells such as: metastable morphology and heating ${ }^{32}$, oxygen and water ${ }^{33}$, and irradiation ${ }^{34 ; 35}$. Indeed, the degradation of $\pi$-conjugated molecules is a bottleneck impeding their application in OPV devices ${ }^{36}$. The use of these molecules as device materials in OPV technologies therefore requires the development of an innovative technique that can overcome this degradation problem. An efficient technique consists in encapsulating $\pi$-conjugated molecules inside the hollow core of nanotubes ${ }^{37}$. This strategy is among the best methods to combine the physical properties of both systems without any mechanical or chemical damaging ${ }^{38 ; 39 ; 40}$ and offers opportunities in achieving high efficiency and high stability of organic solar cells towards future industrial manufacture. With this perspective, the encapsulation of CNTs with photoactive molecules would have a major impact on OPV technology particularly thanks to the scaling-up of a variety of deposition and patterning methods available today allowing encapsulate the conjugated polymers inside CNTs $41 ; 42$

In this regard for important feedback on the PCE optimization, we report a reliable computational method to correlate chirality and diameter of SWCNT with characteristics of a 
photoactive nanocomposite. In this context, a non-covalent functionalization is employed and we choose the 4T@SWCNT as model system where the $4 \mathrm{~T}$ is encapsulated into a SWCNT (Figure 1). We developed an hybrid approach, combining the density functional theory and molecular mechanics, to build the dynamical model of 4T@SWCNT. For this hybrid system, we performed total energy minimization to derive a stable structure with an optimal tube diameter. The knowledge of this structure allows to estimate the exciton separation. We also calculated the Raman spectra, using the bond polarizability model combined with the spectral moments method, of the free 4T molecule, empty SWCNTs with different diameters and chiralities, and 4T@SWCNT to analyze the influence of the encapsulation on the Raman fingerprints. From these Raman calculations, we discuss on an eventual charge transfer between the $4 \mathrm{~T}$ oligomer and the nanotube.

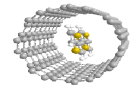

FIGURE 1 Schematic representation of the 4T encapsulation into a SWCNT. The yellow color indicates the Sulfur, grey the Carbon, and white the Hydrogen atoms.

\section{2 | MODEL, METHOD AND AP- PROACH}

Calculation of Raman spectra requires the knowledge of the polarizability tensor (mainly for the intensity of Raman lines) and the dynamical matrix (mainly for the frequency position of Raman lines) of the system.

The dynamical matrix of a system has a $3 \mathrm{Nx} 3 \mathrm{~N}$ dimension, where $\mathrm{N}$ is the number of atoms in the primitive unit cell. Taking the symmetry of the system into account significantly decrease the number of matrix elements to be calculated.
However, in the case of the 4T@ SWCNT hybrid system, we have no symmetry elements, except the trivial identity operation, and all the elements of the dynamical matrix have to be calclated. Fortunately, we can work with a lower or upper triangular matrix because the dynamical matrix is hermitian by definition.

First principles based methods are very accurate to compute the dynamical matrix (frozen phonon or density functional perturbation theory) but their computational cost is very high for large system (like 4T@SWCNT) and especially without any symmetry. Molecular mechanics could be an excellent alternative to build the 4T@SWCNT dynamical matrix but such a force field still remains unknown. Thus, we adopted three-step methodology taking advantage of the densiy functional theory and the molecular mechanics. The idea consists to decompose the 4T@SWCNT hybrid system into two independent subsystems (SWCNT and 4T), which will be coupled by an adequate empirical intermolecular potential. The dynamical matrix of the oligomers molecules will then be calculated using DFT (first step) and the dynamical matrix of SWCNT will be computed from the force constant model introducted by Saito et $\mathrm{al}^{43}$ (second step). The interactions between 4T-SWCNT will be described by a van der Waals potential (third step). We choose a Lennard Jones type:

$$
U_{L J}(r)=4 \varepsilon\left[\left(\frac{\sigma}{r}\right)^{12}-\left(\frac{\sigma}{r}\right)^{6}\right]
$$

with parameters: $\epsilon=2.964 \mathrm{meV}$ and $\sigma=0.3407 \mathrm{~nm}$ for carbon atoms. $\epsilon=10.7 \mathrm{meV}$ and $\sigma=0.3560 \mathrm{~nm}$ for sulfur atoms. $\epsilon=0.67 \mathrm{meV}$ and $\sigma=0.1069 \mathrm{~nm}$ for hydrogen atoms. The Carbon-Sulfur and Hydrogen-Carbon parameters were calculated using Lorentz -Berthelot rule: $\varepsilon_{A B}=\sqrt{\varepsilon_{A} \varepsilon_{B}}$ et $\sigma_{A B}=\left(\sigma_{A}+\sigma_{B}\right) / 2$.

The DFT calculations on 4T molecule have been performed with the SIESTA package, which use pseudo-potentials and localized atomic basis ${ }^{44}$. We used the local density approximation (LDA). The valence electrons were described by a double- $\zeta$ singly polarized basis set. The localization of the basis is controlled by an energy shift of $50 \mathrm{meV}$ and the real space integration was performed on a regular grid corresponding to a plane wave cutoff around 360 Ry. We considered a vaccum size of $12 \AA$ to avoid interactions between adjacent systems. 
The Raman susceptibilty of phonon modes is calculated according to the bond polarizability model of SWCNTs ${ }^{45}$ combined with the one of free $4 \mathrm{~T}$ molecules ${ }^{46}$. This approach has been successfully used to predict the nonresonant Raman spectra of SWCNTs and double-walled carbon nanotubes (DWCNTs) as a function of their lenghts $47 ; 48 ; 49 ; 50$.

Calculations of Raman spectra of our studied systems are based on the spectral moment's method. For more details on this method, see Ref. ${ }^{51}$.

\section{3 | RESULTS}

\section{1 | Stability of 4T@SWCNT}

We determined the range of tube diameters in which the 4T@SWCNT hybrid system is stable. Such a study can guide experimentalists as they provide reliable estimates about reasonable nanotube sizes and the steric hindrance of the $4 \mathrm{~T}$ oligomer inside the tube. We assume in our calculations that the $4 \mathrm{~T}$ size is strongly reduced under nanoconfinement by aligning all the thiophene rings to form a planar structure. The optimized configuration corresponds to an isolated chain inserted into the carbon nanotube and centred along the nanotube main axis. In practice, the structure relaxation was achieved by minimizing the distances between atoms associated to Lennard-Jones potential. Figure 2 shows the Lennard Jones potential for 4T@SWCNT as a function of the tube diameter ranging from 0.8 to $2.5 \mathrm{~nm}$. We observe a minimum at $0.94 \mathrm{~nm}$ corresponding to the equilibrium distance between the SWCNT and 4T molecule. Thus, the insertion of a single 4T oligomer, aligned along the axis of the carbon nanotube and located at its center, is favorable for SWCNTs whose diameter is close to $0.94 \mathrm{~nm}$. This diameter is in agreement with the experimental observations of Alvarez et al. ${ }^{52}$ for a single $4 \mathrm{~T}$ molecule. Note that a distribution of SWCNTs ranging from 0.9 to $1.0 \mathrm{~nm}$ in diameter with corresponding $\mathrm{S} 1$ optical bandgaps ranging from 1.0 to $1.2 \mathrm{eV}^{16 ; 53 ; 54}$, could be employed to capture photons of energy $h \nu>1 \mathrm{eV}$, which is almost the same spectral range captured by a silicon solar cell. Indeed, on the concept of $\mathrm{BHJ}$, following photon absorption of either $4 \mathrm{~T}$ or SWCNT, the excitation of 4T followed by electron transfer, or the excitation of SWCNT followed by hole transfer lead to the same charge separation state, and therefore, the spectral coverage realized by a particular mixture of SWCNTs with diameter around $0.94 \mathrm{~nm}$ can be exploited to optimize lightharvesting and, by extension, the power conversion efficiency of organic solar cells (OSCs).

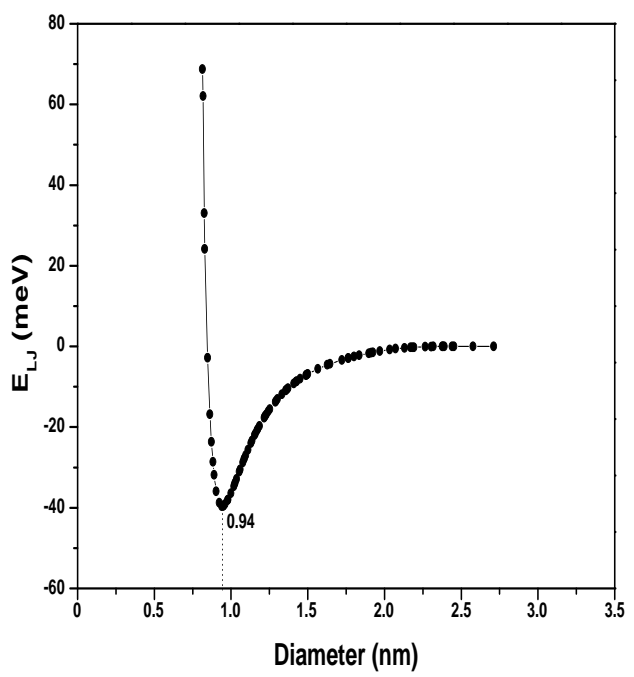

F I G URE 2 Minimization energy calculations of 4T@SWCNT with different diameters.

\section{2 | Raman spectra of isolated 4T}

The calculated Raman spectrum of $4 \mathrm{~T}$ oligomer is reported in Figure 3 up to $1600 \mathrm{~cm}^{-1}$ and shows several distinct lines at 8, 20, 97,160, 315, 369, 463, 633, 697, 844, 877, 1055,1201, $1266,1283,1344,1412,1503,1521$ and $1575 \mathrm{~cm}^{-1}$. We assigned these lines according to the various experimental and theoretical Raman studies on polythiophene reported in literature $55 ; 56 ; 57$. The couple of lines at 8 and $20 \mathrm{~cm}^{-1}$ are libration modes. The lines at 97 and $315 \mathrm{~cm}^{-1}$ are assigned to in-plane vibrations characterized by the C-S-C bending modes (the ring deformation $\mathrm{C}-\mathrm{S}-\mathrm{C}$ ). The in-plane vibrations characterized by torsional modes S-C are evidenced at 160 $\mathrm{cm}^{-1}$. The line at $369 \mathrm{~cm}^{-1}$ is assigned to in-plane vibrations characterized by torsional modes $\mathrm{C}-\mathrm{C}$ and out-of-plane vibrations characterized by torsional modes S-C. The line at 463 
$\mathrm{cm}^{-1}$ is a torsion around the S-C bond. The lines at 633 and $697 \mathrm{~cm}^{-1}$ are assigned to in-plane C-S-C bending. The C-S stretching modes contribute to the line at $844 \mathrm{~cm}^{-1}$. The pyramidalizations of the carbon atoms is also probably assigned to this line. The feature at $877 \mathrm{~cm}^{-1}$ is associated to in-plane $\mathrm{C}-\mathrm{C}-\mathrm{C}$ bending modes. The $\mathrm{C}-\mathrm{C}$ stretching modes along with the $\mathrm{C}-\mathrm{C}-\mathrm{H}$ bendings give rise to the line observed at 1055 $\mathrm{cm}^{-1}$. The modes at $1201 \mathrm{~cm}^{-1}$, along with those at 1266 , 1283 and $1344 \mathrm{~cm}^{-1}$ are assigned to a progression of the C$\mathrm{C}-\mathrm{H}$ bending modes with contributions of the inter-ring C-C stretching modes. Finally, lines centered in the 1400-1600 $\mathrm{cm}^{-1}$ range are assigned to $\mathrm{C}-\mathrm{C}$ stretching modes.

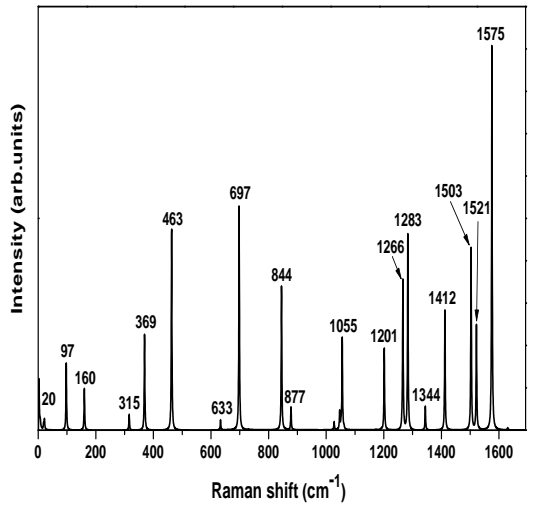

F IGURE 3 Calculated Raman spectrum of a 4T molecule.

\subsection{Raman spectra of 4T@SWCNT}

Raman lines of pristine (empty) carbon nanotubes can be divided into two ranges of frequencies: below $400 \mathrm{~cm}^{-1}$ where are located the radial breathing modes (RBM) and above 1500 $\mathrm{cm}^{-1}$ where are located the tangential modes (G-band). These modes are especially relevant to probe an eventual charge transfer between 4T and SWCNT. This possibility (or not) of a charge transfer is of major interest in the nanotube community workers and constitutes the main question of this work. To answer to this question, we considered six 4T@SWCNT with different tube chirality $(n, m)$ and whose the diameters are around $0.94 \mathrm{~nm}$ (the optimal value found in section 3.1 to encapsulate only one 4T). Among them, three are metallic $[(12,0),(11,2)$ and $(7,7)]$ and three are semiconducting $[(8,6),(9,5)$ and $(10,3)]$. The ZZ-polarized Raman spectra of empty SWCNTs and hybrid systems after minimizations are displayed in Figures 4 (metallic tubes) and 5 (semiconducting tubes). For each SWCNT and 4T@SWCNT, the intensities of the different spectra are normalized and can be therefore compared.

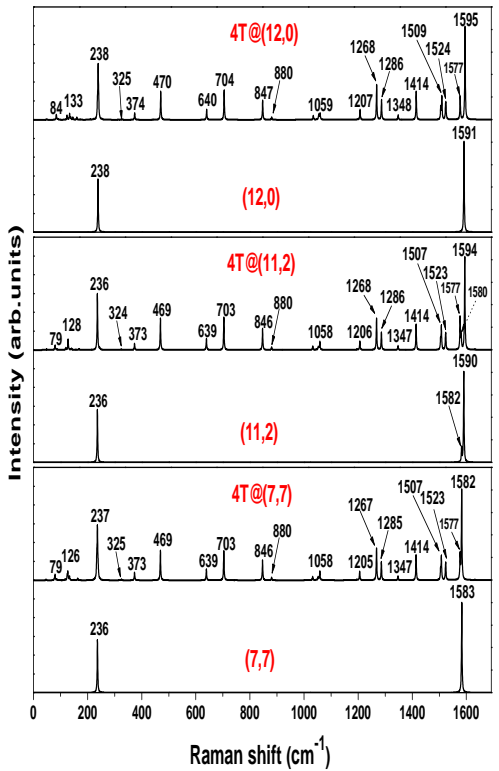

F I G URE 4 Calculated Raman spectra of a 4T encapsulated into a metallic SWCNT.

At first glance and whatever the nature (metallic or semiconducting) of the nanotube, Raman spectra of the 4T@SWCNT hybrid systems seem to be to the superposition of the 4T and SWCNT spectra. However, a more careful analysis shows several differences which are characteristic of the interactions between the 4T and SWCNT in hybrid systems. Indeed, the libration modes $\left(8\right.$ and $\left.20 \mathrm{~cm}^{-1}\right)$ of $4 \mathrm{~T}$ oligomer and evidenced in Figure 3 disappear in Figures 4 and 5 after encapsulation. This type of reciprocating motion, in which the free $4 \mathrm{~T}$ repeatedly rotates slightly back and forth, is deactivated after encapsulation. This absence of libration can explains a certain stability of $4 \mathrm{~T}$ inside the SWCNT cavity. 
In addition, an upshift of all Raman lines between 1 and 9 $\mathrm{cm}^{-1}$ is observed in the 4T@SWCNT spectra with respect to corresponding ones in the free $4 \mathrm{~T}$ oligomer. This upshift associated to van der Waals interaction is another hint of a significant interaction between $4 \mathrm{~T}$ and the nanotubes.

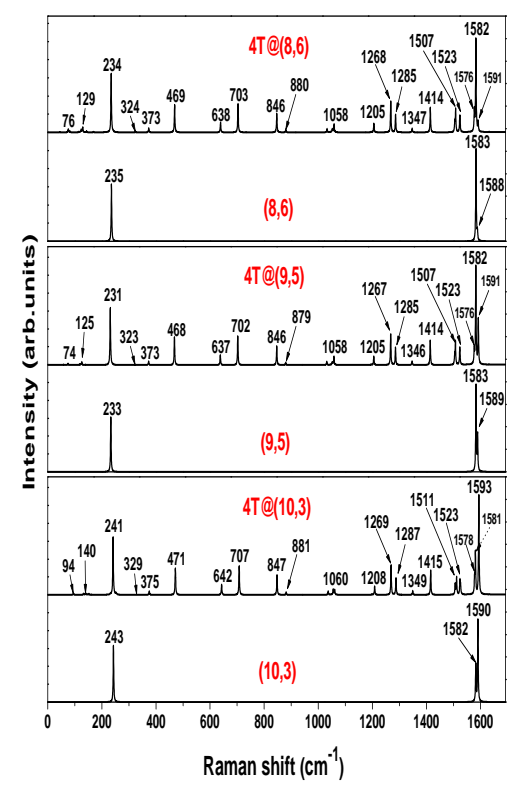

F I G URE 5 Calculated Raman spectra of a 4T encapsulated into a semiconducting SWCNT.

The radial breathing modes (RBM) clearly exhibit a frequency upshift after encapsulation by about $1 \mathrm{~cm}^{-1}$ for 4T@(7,7) nanotube (Figure 6, bottom). Let us recall that, charge transfer (positive or negative) onto individual metallic nanotubes has been shown to induce a small RBM up-shift of about $2 \mathrm{~cm}^{-158}$. The shift of a few to a higher frequency indicates that the diameter of the nanotube is energetically favorable for the encapsulated molecules as predicted by Okada and co-workers for nanotubes encapsulating fullerenes ${ }^{59}$. For 4T@(11,2) and 4T@(12,0) hybrid systems, no shift of RBM position is observed in Figure 6. Such a behavior is observed when rubidium atoms (electron donor molecule) are encapsulated into bundles of $\mathrm{CNT}^{60}$. Conversely, in the case of semiconducting nanotubes, the RBM observed in empty tubes is downshifted by about $2 \mathrm{~cm}^{-1}$ for both 4T@(10,3) and $4 \mathrm{~T} @(9,5)$ and by about $1 \mathrm{~cm}^{-1}$ for $4 \mathrm{~T} @(8,6)$ as shown in figure 6. In order to discuss the origin of this mode softening, it is worth mentioning that the dispersive interactions (vdW) lead to a small downshift $\left(2\right.$ or $\left.3 \mathrm{~cm}^{-1}\right)$ for fullerene molecules 61 .
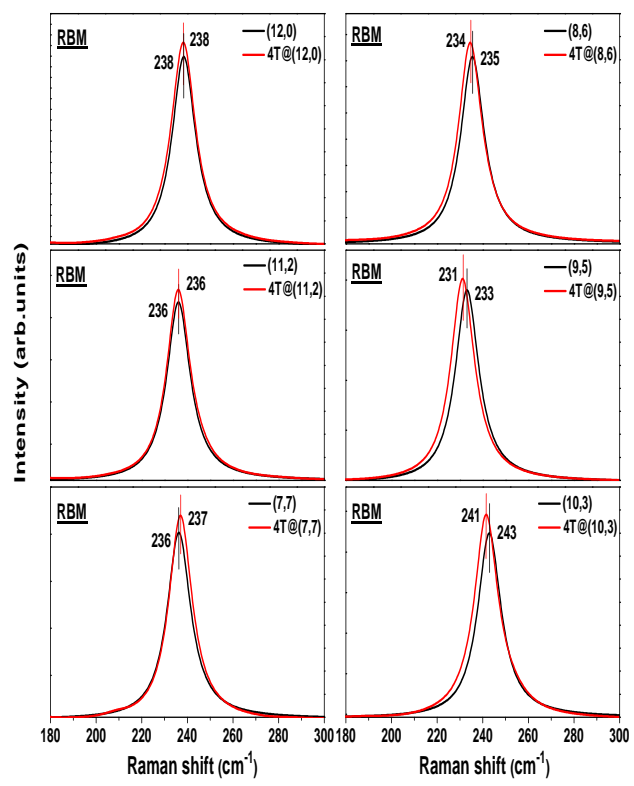

F I G U RE 6 Calculated Raman spectra of empty SWCNT (black curve) and 4T@SWCNT (red curve) in the RBM range. (left) Metallic SWCNT. (right) Semiconducting SWCNT.

Generally, the RBM shifts are at most $-3 \mathrm{~cm}^{-1}$ for dispersive interactions ${ }^{59 ; 61 ; 62}$ and $2 \mathrm{~cm}^{-1}$ for charge transfer ${ }^{58 ; 63}$. In addition, the lower shift of the RBM frequencies originates from modulation of the charge distribution of $\pi$-electrons in the nanotubes induced by the encapsulation ${ }^{59}$. By contrast, insertion of lithium (electron donor molecule) gives rise to a small down-shift ${ }^{64}$. However, the physical interactions of thiophene molecules with the nanotubes are probably dispersive and combined to a slight charge transfer as reported by Alvarez et $a l^{52}$. using Raman and infrared spectroscopies.

Figure 7 displays the calculated ZZ-polarized Raman spectra within the G-band range $\left(1560-1620 \mathrm{~cm}^{-1}\right)$ of the empty SWCNT (black curves) with different chirality. For the $(12,0)$ zigzag tube (chiral angle $\theta=0^{\circ}$ ) and the $(7,7)$ armchair (chiral angle $\theta=30^{\circ}$ ) tubes, the spectra are featured by a single 


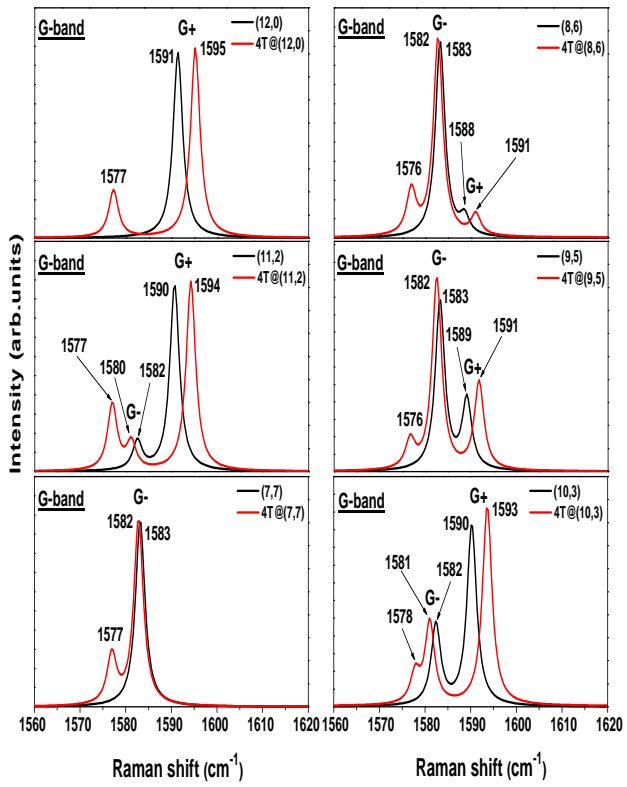

F I G U RE 7 The G-band Raman spectra of empty SWCNT (black curve) and 4T@SWCNT (red curve). (left) Metallic SWCNT. (right) Semiconducting SWCNT.

peak assigned to $\mathrm{A}_{1 \mathrm{~g}}(\mathrm{ZZ})$ tangential modes. This peak is centered at $1582 \mathrm{~cm}^{-1}$ in the $(7,7)$ SWCNT and at $1591 \mathrm{~cm}^{-1}$ in the $(12,0)$ SWCNT. In the following, we use the terms $\mathrm{G}^{-}$ and $\mathrm{G}^{+}$when referring to the lower-frequency peak and the higher-frequency peak, respectively. When increasing $\theta$ from 0 to $30^{\circ}$, we observe the appearance of two peaks $\left(\mathrm{G}^{-}\right.$and $\mathrm{G}^{+}$) at frequencies close to the frequency of the zigzag and armchair tangential modes (see for instance the $(11,2)$ whose the chiral angle is $8^{\circ}$ ). Consequently, the $\mathrm{G}^{+}$(resp. $\mathrm{G}^{-}$) peak is dominant in the zigzag (resp. armchair) tube, while both the $\mathrm{G}^{+}$and $\mathrm{G}^{-}$peaks appear in chiral tubes $\left(0^{\circ}<\theta<30^{\circ}\right)$. The intensity of these peaks $\left(\mathrm{G}^{-}\right.$and $\left.\mathrm{G}^{+}\right)$shifts from the one located around the frequency of the zigzag to that located around the frequency of the armchair when increasing $\theta$. In all our calculations, the $\mathrm{G}^{-}$and $\mathrm{G}^{+}$features were assigned to the transverse optical (TO) and the longitudinal optical (LO) phonon modes, respectively, independent of whether the nanotube is metallic or semiconducting. With the $4 \mathrm{~T}$ encapsulation, the 4T@SWCNT spectra (red curves) exhibit not only the G-bands of the nanotubes $\left(\mathrm{G}^{-}\right.$and $\left.\mathrm{G}^{+}\right)$between $1560-1620 \mathrm{~cm}^{-1}$ ) but also the C-C stretching vibrations of the confined 4T molecule located around $1575 \mathrm{~cm}^{-1}$. For metallic SWCNT (left hand part of the figure), the influence of the confinement shows that the $\mathrm{G}^{-}$peak (TO mode) position shows a downshift by $1 \mathrm{~cm}^{-1}$ in the $4 \mathrm{~T} @(7,7)$, the $\mathrm{G}^{+}$peak (LO mode) shows a significant upshift by $4 \mathrm{~cm}^{-1}$ in the $4 \mathrm{~T} @(12,0)$, and the 4T@(11,2) spectrum clearly exhibits the same shift of these two G-bands. For semiconducting SWCNT (right hand part of Figure 7), the three considered tubes, $(10,3),(9,5)$ and $(8,6)$, are chiral with $\theta \sim 13,20$ and $25^{\circ}$, respectively. Both the $\mathrm{G}^{+}$and $\mathrm{G}^{-}$peaks appear in the Raman spectra of empty tubes. After encapsulation, all spectra exhibit a $\mathrm{G}^{-}$peaks (TO modes) downshifted by $1 \mathrm{~cm}^{-1}$. At the opposite, the $\mathrm{G}^{+}$ peaks (LO modes) shows a significant upshift by $3 \mathrm{~cm}^{-1}$ for both the 4T@(10,3) and 4T@(8,6), and by $2 \mathrm{~cm}^{-1}$ for the 4T@(9,5). Based on these observations, The G-band behavior for both metallic and semiconducting SWCNT show similar trends under the confinement effect. The $\mathrm{G}^{-}$(TO-modes) of SWCNT show a slight downshift of about $1 \mathrm{~cm}^{-1}$, whereas the $\mathrm{G}^{+}$(LO-modes) demonstrate a larger upshift between 2 and $4 \mathrm{~cm}^{-1}$. These shifts to higher frequencies of the $\mathrm{G}^{+}$peaks, which corresppond to axial displacement of the atoms (see Figure 8(bottom)), indicate a possible charge transfer from the SWCNT to the $4 \mathrm{~T}$ molecule.

Indeed, the literature reports that p-doping always leads to the contraction of bonds and phonon stiffening ${ }^{65}$. In the other hand, for the slight downshift of the $\mathrm{G}^{-}$peaks, which refers to a circumferential displacement of the atoms (see Figure 8 (top)), there is probably a competition between dispersive interactions of $4 \mathrm{~T}$ molecules with the nanotubes which cause phonon softening and the downshift of the $\mathrm{G}^{-}$peaks, and the effect of slight charge transfer which results in the upshift of the $\mathrm{G}^{-}$peaks. Both effects may compensate each other, and the shift of the G-band is expected to be smaller or even to have the opposite sign than for p-doping.

\section{4 | 4. DISCUSSION}

To make a comparison with the experimental data as realistic as possible, we discuss in this section the result of our calculations on the $\mathrm{G}^{+}$and $\mathrm{G}^{-}$peaks to those obtained over the years by various theoretical and experimental works.

One method to distinguish between metallic and semi- 


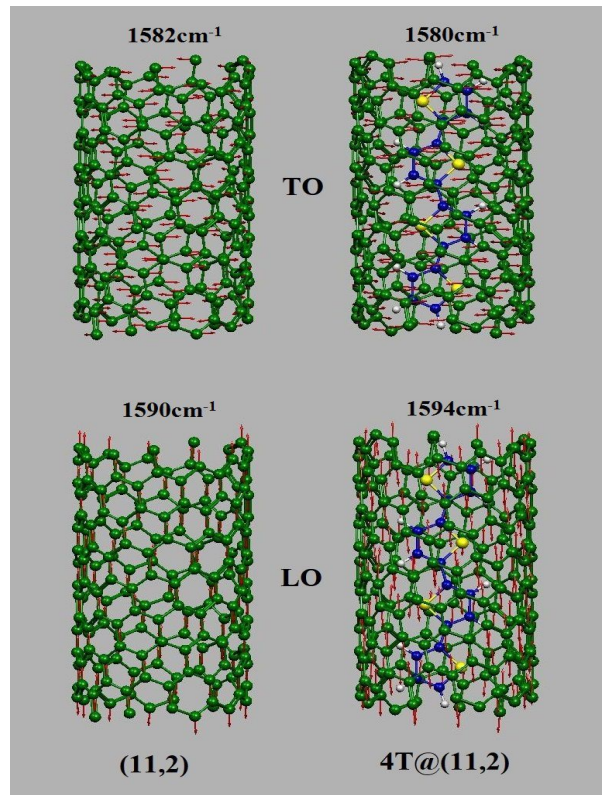

F I G URE 8 Calculated eigen displacement vectors for the tangential modes in $(11,2)$ tube $($ left $)$ and $4 \mathrm{~T} @(11,2)$ (right).

conducting SWCNT is based on the distinct differences in the Raman line shape of the tangential G-band. The Gband of SWCNT consists of two main peaks due to the curvature-induced inequality of the two bond-displacement directions: a lower-frequency peak $\left(\mathrm{G}^{-}\right)$in the $1550-1570$ $\mathrm{cm}^{-1}$ range and a higher-frequency peak $\left(\mathrm{G}^{+}\right)$near 1580 $1590 \mathrm{~cm}^{-1}$. In semiconducting tubes both peaks are sharp (narrow Lorentzian peaks), while in metallic tubes the $\mathrm{G}^{-}$is extremely broad $66 ; 67 ; 68$. However, optical phonon modes in nanotubes were studied theoretically by various methods, such as zone-folding schemes ${ }^{69}$, force-constant models ${ }^{70 ; 71}$ and first-principles based methods $72 ; 73 ; 74 ; 75 ; 76 ; 77 ; 78 ; 79$, and different models have been developed for the description of the peculiar line shape of the Raman G-band in metallic carbon nanotubes. In refs. ${ }^{80}$ for example, Kempa proposed phononplasmon interactions as the origin of the metallic line shape where the $\mathrm{G}^{-}$and $\mathrm{G}^{+}$features were respectively assigned to the TO and LO phonon as in the case of semiconducting tubes. In contrast, other models treated this broad $\mathrm{G}^{-}$peak through a Breit-Wigner-Fano (BWF) line shape ascribed to the strong coupling between the LO phonon and electronic excitations ${ }^{68 ; 72 ; 81 ; 82}$. In these models, the LO frequency is below the TO frequency due to the strong electron-phonon coupling, and consequently, the $\mathrm{G}^{-}$peak is assigned to the LO phonon. As a result, the frequency of the LO phonon drops below the frequency of the TO phonon in metallic nanotubes. However, the assignment of the $\mathrm{G}^{-}$and $\mathrm{G}^{+}$Raman peaks to the TO and LO phonons for metallic nanotubes is still under debate with contradicting conclusions appearing in the literature, even if there is accumulating consensus that the broad $\mathrm{G}^{-}$peak is a softened and broadened LO phonon feature arising from Kohn anomalies.

In view of these results from the literature, the splitting of the $\mathrm{G}^{-}$and $\mathrm{G}^{+}$peaks (two narrow Lorentzian peaks) in our calculations, and their assignment to the TO and LO phonons respectively, seems to be in good agreement with the experimental data for semiconducting nanotubes. In the case of metallic nanotube, our results are more problematic as the asymmetric G-band line-shape in Raman spectra is related to Fano resonance (BWF) lines. However we highlight that our calculations does not consider the eletron-phonon coupling responsible for the BWF line shape in metallic SWCNT. This will be studied in a future works towards improving the detailed comparison of the theoretical and experimental frequency shifts in hybrid systems with metallic SWCNT. Nevertheless, even with the present limitations, our predictions concerning the number and frequency of Raman lines obtained in the framework of a nonresonant Raman theory do not depend on the resonant process of Raman scattering and can be helpful to interpret the frequencies shifts in experimental data of hybrid systems.

\section{5 | 5. CONCLUSIONS}

In this study, we use an orignal approach to study the encapsulation of a 4T molecule inside SWCNT. The dynamical model of the $4 \mathrm{~T}$ and the nanotube have been respectively described by DFT and molecular mechanics whereas the coupling between the 4T molecule and the nanotube have been described by a Lennard-Jones potential. First, we found that the configurations energetically favorable for the insertion of a single molecule aligned along the nanotube main axis and located at its center, are those with a tube diameter around $0.94 \mathrm{~nm}$. 
Then, for the obtained configurations of 4T@SWCNT hybrid systems with metallic and semiconducting nanotubes, the nonresonant Raman spectra have been calculated using the bond-polarizability model combined with the spectral moments method. The charge transfer in the 4T@SWCNT has been investigated by analysing the Raman-active modes of the 4T molecule, as the RBM and G-band modes of SWCNT, before and after encapsulation. Finally, we conclude that a possible charge transfer between the $4 \mathrm{~T}$ and the SWCNTs independently of their metallic or semiconducting character. In this context, the encapsulation of organic molecules inside carbon nanotube, thus creating a new hybrid system, appear to be of great interest for solar cell applications.

\section{ACKNOWLEDGMENT}

The work was supported by Moulay Ismail University Research Support (13-16) and CONICYT Posdoctodado $2020 \mathrm{~N}^{\mathrm{o}}$ 3200046. JML acknowledges support from Financiamiento basal para centros científicos y tecnológicos de excelencia AFB180001.

\section{CONFLICT OF INTEREST}

You may be asked to provide a conflict of interest statement during the submission process. Please check the journal's author guidelines for details on what to include in this section. Please ensure you liaise with all co-authors to confirm agreement with the final statement.

\section{REFERENCES}

1. Long Ye, Shaoqing Zhang, Lijun Huo, Maojie Zhang, Jianhui Hou, Accounts of chemical research 2014, 47 (5), 15951603.

2. Xiaozhou Che, Yongxi Li, Yue Qu, Stephen R Forrest, $N a$ ture Energy 2018, 3 (5), 422-427.

3. Yong Cui, Huifeng Yao, Ling Hong, Tao Zhang, Ye Xu, Kaihu Xian, Bowei Gao, Jinzhao Qin, Jianqi Zhang, Zhixiang Wei, et al., Advanced Materials 2019, 31 (14), 1808356.

4. Yong Cui, Huifeng Yao, Jianqi Zhang, Tao Zhang, Yuming Wang, Ling Hong, Kaihu Xian, Bowei Xu, Shaoqing Zhang, Jing Peng, et al., Nature communications 2019, 10 (1), 1-8.
5. Lingxian Meng, Yamin Zhang, Xiangjian Wan, Chenxi Li, Xin Zhang, Yanbo Wang, Xin Ke, Zuo Xiao, Liming Ding, Ruoxi Xia, et al., Science 2018, 361 (6407), 1094-1098.

6. Gilles Dennler, Markus C Scharber, Christoph J Brabec, Advanced materials 2009, 21 (13), 1323-1338.

7. Chaohua Cui, Yaowen Li, Yongfang Li, Advanced Energy Materials 2017, 7 (10), 1601251.

8. Yongye Liang, Zheng Xu, Jiangbin Xia, Szu-Ting Tsai, Yue Wu, Gang Li, Claire Ray, Luping Yu, Advanced materials 2010, 22 (20), E135-E138.

9. Martin A Green, Keith Emery, Yoshihiro Hishikawa, Wilhelm Warta, Ewan D Dunlop, Progress in photovoltaics: research and applications 2015, 23 (1), 1-9.

10. Yuze Lin, Zhi-Guo Zhang, Huitao Bai, Jiayu Wang, Yuehan Yao, Yongfang Li, Daoben Zhu, Xiaowei Zhan, Energy \& Environmental Science 2015, 8 (2), 610-616.

11. Yunpeng Qin, Mohammad Afsar Uddin, Yu Chen, Bomee Jang, Kang Zhao, Zhong Zheng, Runnan Yu, Tae Joo Shin, Han Young Woo, Jianhui Hou, Advanced Materials 2016, 28 (42), 9416-9422.

12. Wenchao Zhao, Deping Qian, Shaoqing Zhang, Sunsun Li, Olle Inganäs, Feng Gao, Jianhui Hou, Advanced materials 2016, 28 (23), 4734-4739.

13. Jingbo Zhao, Yunke Li, Guofang Yang, Kui Jiang, Haoran Lin, Harald Ade, Wei Ma, He Yan, Nature Energy 2016, 1 (2), 1-7.

14. Andrew J Ferguson, Jeffrey L Blackburn, Nikos Kopidakis, Materials Letters 2013, 90, 115-125.

15. Qiuhong Zhang, Zongwu Bai, Feng Du, Liming Dai in Nanotube Superfiber Materials, Elsevier, 2019, pp. 695-728.

16. Hiromichi Kataura, Y Kumazawa, Y Maniwa, I Umezu, S Suzuki, Yo Ohtsuka, Y Achiba, Synthetic metals 1999, 103 (1-3), 2555-2558.

17. T Dürkop, SA Getty, Enrique Cobas, MS Fuhrer, Nano letters 2004, 4 (1), 35-39.

18. Ali Javey, Jing Guo, Qian Wang, Mark Lundstrom, Hongjie Dai, nature 2003, 424 (6949), 654-657.

19. Anusorn Kongkanand, Prashant V Kamat, ACS nano 2007, $1(1), 13-21$.

20. Xuqiang Ji, Yuanhong Xu, Wenling Zhang, Liang Cui, Jingquan Liu, Composites Part A: Applied Science and Manufacturing 2016, 87, 29-45. 
21. G Alvarado-Tenorio, HJ Cortina-Marrero, ME Nicho, PA Márquez Aguilar, H Hu, Materials Science in Semiconductor Processing 2016, 56, 37-42.

22. Jeffrey L Blackburn, ACS Energy Letters 2017, 2 (7), 15981613.

23. T Hosseini, N Kouklin, Carbon Nanotubes: Current Progress of their Polymer Composites 2016, 95.

24. Livia Noëmi Glanzmann, Duncan John Mowbray, Diana Gisell Figueroa del Valle, Francesco Scotognella, Guglielmo Lanzani, Angel Rubio, The Journal of Physical Chemistry C 2016, 120 (3), 1926-1935.

25. Emmanuel Kymakis, Gehan AJ Amaratunga, Reviews on advanced materials science 2005, 10 (4), 300-305.

26. Carla D Canestraro, Mariane C Schnitzler, Aldo JG Zarbin, MGE Da Luz, Lucimara S Roman, Applied surface science 2006, 252 (15), 5575-5578.

27. Takeshi Tanaka, Hehua Jin, Yasumitsu Miyata, Shunjiro Fujii, Hiroshi Suga, Yasuhisa Naitoh, Takeo Minari, Tetsuhiko Miyadera, Kazuhito Tsukagoshi, Hiromichi Kataura, Nano Letters 2009, 9 (4), 1497-1500.

28. Ralph Krupke, Frank Hennrich, Hilbert v Löhneysen, Manfred M Kappes, Science 2003, 301 (5631), 344-347.

29. Yosuke Kanai, Jeffrey C Grossman, Nano letters 2008, 8 (3), 908-912.

30. Josh M Holt, Andrew J Ferguson, Nikos Kopidakis, Brian A Larsen, Justin Bult, Garry Rumbles, Jeffrey L Blackburn, Nano letters 2010, 10 (11), 4627-4633.

31. Sebastiano Cataldo, Patrizio Salice, Enzo Menna, Bruno Pignataro, Energy \& Environmental Science 2012, 5 (3), 5919-5940.

32. Christoph J Schaffer, Claudia M Palumbiny, Martin A Niedermeier, Christian Jendrzejewski, Gonzalo Santoro, Stephan V Roth, Peter Müller-Buschbaum, Advanced materials 2013, 25 (46), 6760-6764.

33. Andrew J Parnell, Ashley J Cadby, Alan DF Dunbar, George L Roberts, Alex Plumridge, Robert M Dalgliesh, Maximilian WA Skoda, Richard AL Jones, Journal of Polymer Science Part B: Polymer Physics 2016, 54 (2), 141-146.

34. Laura Corcoles, Jose Abad, Javier Padilla, Antonio Urbina, Solar Energy Materials and Solar Cells 2015, 141, 423428 .

35. Pei Cheng, Xiaowei Zhan, Chemical Society Reviews 2016, 45 (9), 2544-2582.
36. Agnès Rivaton, Sylvain Chambon, Matthieu Manceau, JeanLuc Gardette, Noëlla Lemaître, Stéphane Guillerez, Polymer Degradation and Stability 2010, 95 (3), 278-284.

37. Kazuhiro Yanagi, Yasumitsu Miyata, Hiromichi Kataura, Advanced Materials 2006, 18 (4), 437-441.

38. Taishi Takenobu, Takumi Takano, Masashi Shiraishi, Yousuke Murakami, Masafumi Ata, Hiromichi Kataura, Yohji Achiba, Yoshihiro Iwasa, Nature materials 2003, 2 (10), 683-688.

39. Maria Antonietta Loi, Jia Gao, Fabrizio Cordella, Pascal Blondeau, Enzo Menna, Barbora Bártová, Cécile Hébert, Sorin Lazar, Gianluigi A Botton, Matus Milko, et al., Advanced materials 2010, 22 (14), 1635-1639.

40. Laurent Alvarez, J-L Bantignies, Rozenn Le Parc, Raymond Aznar, J-L Sauvajol, Alexandre Merlen, D Machon, A San Miguel, Physical Review B 2010, 82 (20), 205403.

41. Kenshi Miyaura, Yasumitsu Miyata, Boanerges Thendie, Kazuhiro Yanagi, Ryo Kitaura, Yuta Yamamoto, Shigeo Arai, Hiromichi Kataura, Hisanori Shinohara, Scientific reports 2018, 8 (1), 1-6.

42. Yasuhiro Kinno, Haruka Omachi, Yusuke Nakanishi, Hisanori Shinohara, Chemistry Letters 2018, 47 (8), 10221025.

43. G Dresselhaus, Saito Riichiro, et al., Physical properties of carbon nanotubes, World scientific, 1998

44. José M Soler, Emilio Artacho, Julian D Gale, Alberto García, Javier Junquera, Pablo Ordejón, Daniel Sánchez-Portal, Journal of Physics: Condensed Matter 2002, 14 (11), 2745.

45. S Guha, Jose Menendez, JB Page, GB Adams, Physical Review $B$ 1996, 53 (19), 13106.

46. P Hermet, N Izard, A Rahmani, Ph Ghosez, The Journal of Physical Chemistry B 2006, 110 (49), 24869-24875.

47. A Rahmani, J-L Sauvajol, S Rols, C Benoit, Physical Review B 2002, 66 (12), 125404.

48. A Rahmani, J-L Sauvajol, J Cambedouzou, C Benoit, Physical Review B 2005, 71 (12), 125402.

49. AH Rahmani, M Boutahir, A El Biyaali, B Fakrach, H Chadli, K Sbai, P Hermet, J-L Bantignies, A Rahmani, RSC advances 2016, 6 (47), 41025-41031.

50. Mourad Boutahir, Abdelhai Rahmani, Hassan Chadli, Abdelali Rahmani, The European Physical Journal Applied Physics 2016, 74 (2), 24605. 
51. C Benoit, E Royer, G Poussigue, Journal of Physics: Condensed Matter 1992, 4 (12), 3125.

52. Laurent Alvarez, Yann Almadori, Raul Arenal, R Babaa, Thierry Michel, Rozenn Le Parc, Jean-Louis Bantignies, Bruno Jousselme, Serge Palacin, Patrick Hermet, et al., The Journal of Physical Chemistry C 2011, 115 (24), 11898 11905.

53. Phaedon Avouris, Richard Martel, MRS bulletin 2010, 35 (4), 306-313.

54. Michael S Arnold, Jeffrey L Blackburn, Jared J Crochet, Stephen K Doorn, Juan G Duque, Aditya Mohite, Hagen Telg, Physical Chemistry Chemical Physics 2013, 15 (36), 14896-14918.

55. Alessandra Degli Esposti, Marianna Fanti, Michele Muccini, Carlo Taliani, Giampiero Ruani, The Journal of Chemical Physics 2000, 112 (13), 5957-5969.

56. G Louarn, JP Buisson, S Lefrant, D Fichou, The Journal of Physical Chemistry 1995, 99 (29), 11399-11404.

57. E Agosti, M Rivola, V Hernandez, M Del Zoppo, Giuseppe Zerbi, Synthetic metals 1999, 100 (1), 101-112.

58. Hootan Farhat, Ken-ichi Sasaki, Martin Kalbac, Mario Hofmann, Riichiro Saito, Mildred S Dresselhaus, Jing Kong, Physical review letters 2009, 102 (12), 126804.

59. Susumu Okada, Chemical physics letters 2007, 438 (1-3), $59-62$.

60. N Bendiab, L Spina, A Zahab, P Poncharal, C Marliere, JL Bantignies, E Anglaret, JL Sauvajol, Physical Review B 2001, 63 (15), 153407.

61. David A Britz, Andrei N Khlobystov, Chemical Society Reviews 2006, 35 (7), 637-659.

62. Soon-Kil Joung, Toshiya Okazaki, Naoki Kishi, Susumu Okada, Shunji Bandow, Sumio Iijima, Physical review letters 2009, 103 (2), 027403.

63. Gugang Chen, CA Furtado, S Bandow, S Iijima, PC Eklund, Physical Review B 2005, 71 (4), 045408.

64. N Bendiab, E Anglaret, J-L Bantignies, A Zahab, JL Sauvajol, P Petit, C Mathis, S Lefrant, Physical Review B 2001, 64 (24), 245424

65. Stefan Grimm, Stefan P Schieß1, Yuriy Zakharko, Marcel Rother, Maximilian Brohmann, Jana Zaumseil, Carbon 2017, 118, 261-267.
66. JS Park, K Sasaki, R Saito, W Izumida, M Kalbac, H Farhat, G Dresselhaus, MS Dresselhaus, Physical Review B 2009, $80(8), 081402$

67. Stephen K Doorn, Michael J O'Connell, Lianxi Zheng, Yuntian T Zhu, Shaoming Huang, Jie Liu, Physical review letters 2005, 94 (1), 016802.

68. SDM Brown, A Jorio, a P Corio, MS Dresselhaus, G Dresselhaus, R Saito, K Kneipp, Physical Review B 2001, 63 (15), 155414.

69. RA Jishi, L Venkataraman, MS Dresselhaus, G Dresselhaus, Chemical Physics Letters 1993, 209 (1-2), 77-82.

70. R Saito, T Takeya, T Kimura, G Dresselhaus, MS Dresselhaus, Physical Review B 1998, 57 (7), 4145.

71. J Maultzsch, S Reich, C Thomsen, E Dobardžić, I Milošević, M Damnjanović, Solid state communications 2002, 121 (910), 471-474.

72. O Dubay, G Kresse, H Kuzmany, Physical review letters 2002, 88 (23), 235506.

73. Orest Dubay, Georg Kresse, Physical Review B 2003, 67 (3), 035401.

74. Daniel Sánchez-Portal, Emilio Artacho, José M Soler, Angel Rubio, Pablo Ordejón, Physical Review B 1999, 59 (19), 12678.

75. S Reich, C Thomsen, P Ordejón, Physical Review B 2001, 64 (19), 195416.

76. K-P Bohnen, R Heid, HJ Liu, CT Chan, Physical review letters 2004, 93 (24), 245501.

77. Damien Connétable, G-M Rignanese, J-C Charlier, Xavier Blase, Physical review letters 2005, 94 (1), 015503.

78. Ryan Barnett, Eugene Demler, Efthimios Kaxiras, Physical Review B 2005, 71 (3), 035429.

79. Ivanka Milošević, Edib Dobardžić, Milan Damnjanović, Physical Review B 2005, 72 (8), 085426.

80. K Kempa, Physical Review B 2002, 66 (19), 195406.

81. Michele Lazzeri, Stefano Piscanec, Francesco Mauri, AC Ferrari, J Robertson, Physical review B 2006, 73 (15), 155426.

82. Stefano Piscanec, Michele Lazzeri, John Robertson, Andrea C Ferrari, Francesco Mauri, Physical Review B 2007, 75 (3), 035427 